\title{
L'accompagnement vers la qualité : une source d'inspiration pour les entrepreneurs en phase de pré-création
}

\section{Sarhan Abdennadher}

\section{(2) OpenEdition Journals}

Édition électronique

URL : https://journals.openedition.org/ei/6920

DOI : 10.4000/ei.6920

ISSN : 2553-1891

Éditeur

Association Économie et Institutions

Référence électronique

Sarhan Abdennadher, « L'accompagnement vers la qualité : une source d'inspiration pour les entrepreneurs en phase de pré-création », Économie et institutions [En ligne], 29 | 2021, mis en ligne le 01 décembre 2021, consulté le 02 février 2022. URL : http://journals.openedition.org/ei/6920 ; DOI : https://doi.org/10.4000/ei.6920

Ce document a été généré automatiquement le 2 février 2022.

Revue Économie et institutions 


\title{
L'accompagnement vers la qualité : une source d'inspiration pour les entrepreneurs en phase de pré- création
}

\author{
Sarhan Abdennadher
}

\section{Introduction}

1 Bien que la création d'entreprise soit reconnue comme une activité individuelle, la réalité est généralement bien différente; l'environnement interagit continuellement avec la prise de décision de l'entrepreneur et l'implication des parties prenantes est capitale dans la réussite du projet entrepreneurial avant, pendant et après la création (Verzat et Toutain, 2015). De nos jours, les entrepreneurs et leurs entreprises sont de plus en plus confrontés à subir les effets de la concurrence. Cette dernière, devenue plus acharnée, risque de ternir la réussite de l'aventure entrepreneuriale. Le client, synonyme de chiffre d'affaires, est considéré de sa part comme une des priorités majeures de l'entreprise et se trouve au centre de la réflexion stratégique des entrepreneurs. Certes, pour assurer la réussite de son activité, l'entrepreneur se trouve dans l'obligation de se démarquer de la concurrence en répondant au mieux aux attentes et exigences du marché, en d'autres termes de ses clients.

Désormais, les entrepreneurs qui souhaitent s'installer pour leur propre compte se trouvent face à deux défis majeurs, mais qui vont de soi : la possession d'un avantage concurrentiel et la satisfaction du client. Selon Issor (2017), le but de toute entreprise est de donner satisfaction aux besoins de ses clients en vue d'en tirer des profits; la satisfaction est considérée comme une préoccupation permanente des dirigeants du fait qu'elle constitue un pilier de la pérennité de l'entreprise. Pour cela, les entrepreneurs, afin d'assurer leur prospérité, la survie et la continuité de leurs projets, sont appelés à jouer la carte de la qualité en se conformant à des normes et à un 
système de certification s'ils désirent se maintenir devant la concurrence, satisfaire et fidéliser leurs clients. Hentic-Giliberto et Berger-Douce (2017) ajoutent que l'accompagnement dans les phases précoces du projet entrepreneurial est particulièrement décisif car il peut induire le potentiel de survie et de croissance de l'entreprise. À ce niveau, le rôle des structures d'accompagnement est d'une grande importance en ce qu'elles visent à communiquer aux entrepreneurs des informations relatives à l'environnement institutionnel et économique de l'entreprise, à leur transférer des compétences techniques, à leur fournir plusieurs services, installations et ressources pour soutenir leur aventure entrepreneuriale et les aider à mûrir leurs projets (El Amrani et al., 2016; Mas-Verdú et al., 2015). Qu'il s'agisse du démarrage, du développement ou de la pérennisation de l'activité, les services proposés et l'intervention des structures d'accompagnement doivent être adaptés aux besoins de l'entrepreneur qui se positionne, ainsi, comme demandeur d'une prestation qui permettra le cheminement de son idée afin qu'elle aboutisse à un projet de création viable (Hentic-Giliberto et Berger-Douce, 2017).

3 C'est dans ce contexte que s'inscrit ce travail de recherche. Il a pour objectif d'explorer, à partir de l'observation de douze cas de projets en cours de création, le rôle des structures d'accompagnement dans l'orientation des entrepreneurs en phase de précréation. Ceux-ci, soucieux de l'avenir de leur aventure, sont incités à adopter une démarche qualité afin d'assurer la réussite et la survie de leurs projets. Dominée par les prestations d'accompagnement, de conseil stratégique et de prospection de clientèle (Pluchart, 2014), la phase de pré-création, qui fait intervenir l'accompagnement, la qualité et les entrepreneurs, convient le plus à la question de notre recherche. En Tunisie, à notre connaissance, aucune recherche n'a été réalisée pour traiter cette thématique. En outre, dans la communauté entrepreneuriale, les phases de précréation ont été très peu investiguées (Aouni et Surlemont, 2007).

\section{La création d'entreprises et l'accompagnement vers la qualité}

\subsection{L'importance de l'accompagnement}

4 Pour Gagnon et al. (2011), l'accompagnement d'une personne signifie l'accueillir et l'écouter, connaitre son parcours, ses doutes, mais aussi ses compétences et ses aspirations pour lui permettre de surmonter ses difficultés et ses éventuels échecs. Hernandez (1999) a montré que la démarche stratégique de l'entrepreneur est facilitée par un accompagnement de qualité, et la phase de maturation du processus, durant laquelle l'accompagnement entrepreneurial par des structures extérieures est primordial, permet de vérifier la cohérence entre le créateur et son projet. Julien (2003) présente la création d'une entreprise comme un processus collectif qui commence dans son environnement et est soutenue par la confiance partagée dans des réseaux de proximité stimulés par les acteurs régionaux. Kouada et al. (2018) avancent que l'accompagnement contribue à créer un contexte favorable à l'entrepreneuriat en fournissant aux porteurs de projets un environnement sécurisant en leur offrant un certain nombre de services (locaux, conseils, etc.).

5 Certes, l'accompagnement entrepreneurial offre à l'entrepreneur les atouts nécessaires pour maximiser les chances de réussite de son projet (Fayolle, 2004), et constitue un 
critère majeur optimisateur de succès de l'acte entrepreneurial (Wolff et Cuénoud, 2017). Ben Tahar Gheryani et Boujelbène (2017) ajoutent que l'accompagnement entrepreneurial agit positivement sur la survie des entreprises accompagnées à partir du conseil personnalisé assuré par les accompagnants et de l'effet positif et significatif de la fréquence de contacts effectués. Selon Theodoraki et Messeghem (2016), le principal rôle des structures d'accompagnement est de mettre en relation les porteurs de projets avec les acteurs et les ressources nécessaires pour le développement du projet. Ainsi, l'accompagnement contribue à favoriser la pérennité des entreprises et à en améliorer les performances (Grossetti et Barthe, 2008).

6 Messeghem et al. (2014, p. 20) définissent l'accompagnement entrepreneurial comme : " un processus organisé par une tierce partie, s'inscrivant dans la durée et permettant à un (ou des) entrepreneur(s) de bénéficier d'une dynamique d'apprentissage (formation, conseil, etc.), d'un accès à des ressources (financières, informationnelles, etc.), d'une mise en réseau, de services (administratifs, hébergement, etc.) et d'une aide à la décision (coaching, mentorat, etc.) ». Dans la littérature francophone, l'accompagnement fait référence à la relation interpersonnelle qui existe entre un porteur de projet et son accompagnant et l'apprentissage qui résulte de cette interaction, la littérature anglo-saxonne, de sa part, s'attache à le définir sous l'angle d'une structure organisationnelle ayant développé des compétences managériales permettant de soutenir la création et la croissance d'une entreprise (Messeghem et al., 2014).

7 À travers une analyse sémantique, Paul (2004) distingue trois approches d'accompagnement complétées par Fatien (2008) et Deschamps et al. (2010) :

- Conduire : le maître (l'accompagnant qui sait et qui transmet) exerce un rapport d'autorité sur l'élève (l'accompagné qui dépend de l'accompagnant). Ce dernier reçoit, après diagnostic du projet, des solutions (conseil), des connaissances théoriques et pratiques (formation) et bénéficie de l'expérience du maître (mentorat). À cela s'ajoute un accompagnement à la carte (coaching).

- Guider: l'accompagné est orienté par l'accompagnant, ce qui suppose un échange bidirectionnel dans le cadre d'une co-construction des savoirs. L'accompagnant se place dans une posture empathique de dialogue et d'écoute vis-à-vis de l'accompagné pour lui faciliter le développement individuel afin de trouver «le bon chemin » (counselling). À ce niveau, l'expérience de l'accompagnant (mentorat) et l'accompagnement sur mesure (coaching) aident l'accompagné dans la prise de décision et à forger ses solutions en vue d'atteindre ses objectifs.

- Escorter : l'accompagné est assisté par l'accompagnant, ce qui suppose l'inscription dans une logique de soutien et de transmission des savoirs et des valeurs professionnels (tutorat). L'accompagnant véhicule l'idée de protection, d'attention portée à autrui dans une dynamique de construction où il est impliqué au côté de l'accompagné ; celui qui escorte va protéger, secourir, soutenir, ce qui peut passer par l'accueil ou l'introduction (coaching).

Tableau 1 : sémantiques et pratiques d'accompagnement

\begin{tabular}{|l|l|l|l|l|l|l|l|}
\hline \multicolumn{2}{|c|}{} & \multicolumn{5}{|l|}{ Pratiques d'accompagnement } \\
\cline { 2 - 7 } & & Conseil & Formation & Counselling & Tutorat & Mentorat & Coaching \\
\hline $\begin{array}{l}\text { Sémantiques de } \\
\text { l'accompagnement }\end{array}$ & Conduire & & & & & & \\
\cline { 3 - 7 }
\end{tabular}


8 L'accompagnement est devenu une passerelle obligatoire dans le monde entier car il permet aux entrepreneurs de gagner du temps et leur facilite l'accès aux différentes ressources (Belhaj et al., 2018). Ces ressources, sous forme de conseils stratégiques et de prestations fonctionnelles et opérationnelles (assistance technique, commerciale, juridique, comptable, financière, etc.) (Plane et Torres, 1998) doivent permettre d'établir un profil d'entrepreneur, un diagnostic de son modèle d'affaires et un audit de son plan d'affaires, mais notamment de lui faciliter l'accès à d'autres ressources (partenaires, formation, brevets et marques, équipements et matériels, etc.) afin de contribuer à la construction des avantages concurrentiels de son entreprise (Grossetti et Barthe, 2008 ; Lechner et al., 2006 ; Hoang et Atoncic, 2003).

Pour Degeorge (2017), l'accompagnement entrepreneurial a pour objectifs: le développement des compétences managériales, l'optimisation de l'adéquation homme/ projet, l'orientation et l'aide à la formalisation du business model ou du business plan, l'intégration et la socialisation du projet dans le marché et les réseaux économiques, etc. L'auteur ajoute que l'accompagnement doit dépasser les simples outils habituels pour prendre en compte le caractère contingent, créatif et inductif de l'activité entrepreneuriale. Mhamed Hichri et al. (2017) rejoignent cette idée en insistant sur l'intégration des aptitudes psychosociologiques et des capacités managériales dans l'accompagnement entrepreneurial ce qui peut contribuer à la croissance de l'entreprise. Pouka Pouka et al., (2019) ajoutent que l'accompagnement entrepreneurial offre aux jeunes entreprises des possibilités de croissance sur trois niveaux: l'employabilité, le chiffre d'affaires et la productivité.

De sa côté, Mathot (2010) distingue les huit groupes d'accompagnants suivants: les réseaux institutionnels, les organisations professionnelles, les organismes d'appui des demandeurs d'emploi, les réseaux de financement et d'assurances, les réseaux d'expertise juridique et comptable, les services publics, les foires et salons professionnels et les sites internet. Grossetti et Barthe (2008), quant à eux, distinguent entre les réseaux personnels (famille, amis et relations professionnelles occasionnelles) et les réseaux organisés de Mathot (2010), d'une part, et d'autre part, entre les médiateurs qui conduisent, guident et escortent (Paul, 2004) et les apporteurs de ressources.

Quoiqu'ils soient multiples et différents, l'apport des réseaux d'accompagnement reste tributaire de leur performance. Cette dernière dépend de trois critères: l'efficacité (mesurée par la pérennité et la croissance des entreprises accompagnées), l'efficience (évaluée par la facilité et la rapidité d'accès aux ressources apportées aux créateurs) et l'effectivité (appréciée à partir du niveau de satisfaction des acteurs impliqués) (Paturel, 2000). Messeghem et al., quant à eux, (2010) évaluent les performances des réseaux en fonction des indicateurs de rentabilité (financier), des indicateurs de satisfaction (client), des indicateurs de productivité (processus) et du niveau des connaissances apportées (apprentissage). 


\subsection{L'accompagnement entrepreneurial en Tunisie}

Dans l'optique de créer des conditions favorables à la création d'entreprises, l'État doit choisir d'assumer correctement ses fonctions et mettre en place des dispositifs de formation et de soutien nécessaires pour encourager et soutenir les entrepreneurs (Fortin, 2002). L'État, dont l'action est le facteur qui possède l'influence la plus significative sur l'activité entrepreneuriale (Wilken, 1979), peut collaborer à la structuration d'un milieu, quoique artificiel, propice à la création de nouvelles entreprises créatrices de valeur et de richesse (Zghal, 2004). Au sens de Wilken (1979), seule l'action du gouvernement a le pouvoir et la légitimité pour agir sur les conditions économiques et non-économiques qui, à leur tour, influencent le niveau d'entrepreneuriat. Ainsi, l'État est un partenaire nécessaire et indispensable à la création d'entreprises qui doit y jouer pleinement son rôle en garantissant les systèmes judiciaires et les règles de jeu, misant sur la mobilisation des ressources, offrant un service de qualité aux collectivités en impulsant un développement durable fondé sur la culture entrepreneuriale.

En Tunisie, depuis le début des années 70, l'action entrepreneuriale a connu un essor considérable. Ceci peut être expliqué, d'une part, par le désengagement de l'État de la sphère de la propriété et de l'investissement et, d'autre part, par l'insertion accrue de 1'économie tunisienne dans un système mondial de production ouvert, concurrentiel et innovateur (Chihi, 2005). L'expérience tunisienne en matière d'accompagnement entrepreneurial avait été engagée à travers une politique délibérée visant à stimuler l'initiative privée et favoriser la dynamique de la création d'entreprises. L'encouragement de l'initiative et de la création d'entreprises a débuté surtout à la suite de l'adoption du plan d'ajustement structurel qui a commencé depuis le début des années 1980. Depuis, on assiste à une amélioration continue au niveau du fonctionnement des mécanismes d'aide et d'incitation à la création d'entreprises. Des mesures et des moyens considérables ont été instaurés pour sensibiliser et encourager l'entrepreneuriat afin de créer un contexte environnemental favorable à travers l'octroi de certains avantages fiscaux et financiers et l'instauration d'un environnement institutionnel stimulant: des institutions d'encadrement et de soutien, des facilitations des démarches, etc. (Abdennadher, 2012). En effet, l'État tunisien a créé un environnement institutionnel propice à la naissance d'un paysage entrepreneurial diversifié par la mise en place de plusieurs structures telles que : la banque tunisienne de solidarité, l'agence nationale de l'emploi et du travail indépendant, les pépinières d'entreprises, l'agence de promotion de l'industrie et de l'innovation, etc. (Ben Ali, 2020 ; Haddad et Melliti, 2018 ; Bounouh, 2017 ; Abdennadher, 2012).

\subsection{Le rôle de la qualité}

Pour assurer leur pérennité et continuer à réaliser une croissance durable dans un environnement fortement concurrentiel et qui ne cesse de se métamorphoser, les entreprises se trouvent devant le choix d'adopter diverses stratégies: maitriser les coûts, offrir des produits satisfaisants, anticiper l'évolution du marché, etc. De ce fait, les entreprises sont appelées à différencier leurs offres et positionner leurs produits par rapport à ceux des concurrents dans le but d'obtenir un avantage concurrentiel 
pour pouvoir survivre et se développer. Tout cela ne peut être réalisé qu'à travers l'intégration d'un système qualité digne de ce nom (El Kahri et al., 2014).

Ainsi, l'instauration de la qualité constitue une alternative permettant à l'entreprise d'assurer sa continuité dans un environnement en perpétuel changement. En effet, étant donné le caractère changeant de son environnement, la qualité apparait comme une variable mouvante et protéiforme. Elle est le produit de son contexte, et doit donc être constamment remise en cause. La reconnaissance de la pluralité des formes de l'accord dans la définition de la qualité est l'un des fondements majeurs de l'économie des conventions (Eymard-Duvernay, 1989) qui ont mis en avant le fait que la qualité des biens ne peut se réduire à des critères exclusivement marchands et qu'il existe une pluralité des formes d'accord à prendre en compte (Da Silva, 2018). Pour EymardDuvernay (1995), l'accord sur la qualité est inscrit dans un réseau d'acteurs ouvert, hétérogène et fluctuant.

Devenue comme un des plus importants critères de choix pour les différentes catégories de clients, la qualité est un facteur clés de succès pour les entreprises. Elle se présente comme une variable à travers laquelle les entreprises peuvent répondre aux exigences des clients et satisfaire leurs attentes; "c'est une démarche d'amélioration continue qui vise à améliorer la compétitivité de l'entreprise de manière durable en alliant efficacité et efficience afin de délivrer des produits ou des services répondants aux attentes $d u$ marché et des clients" (DGE et CNS, 2016, p.6). Dans ce sens, il est essentiel pour l'entreprise de connaître convenablement ses bénéficiaires et leurs besoins afin de définir ses objectifs et ses finalités (Delvosalle, 2002).

Certes, la mondialisation, l'ouverture et l'extension des marchés ont poussé les entreprises à raisonner aussi bien à l'échelle nationale qu'internationale. Ce changement en matière de réflexion stratégique a fait apparaitre la qualité comme un facteur de compétitivité pour l'entreprise ; seulement pour jouer pleinement ce rôle qui lui est assigné, la qualité ne doit se limiter au produit, mais elle doit être perçue de façon beaucoup plus large englobant aussi bien le produit, le processus de production ainsi que toute l'organisation (El Kahri et al., 2014). Selon Ruževičius (2013), la qualité se développa jusqu'à ce qu'elle englobe, non seulement les produits de consommation, mais aussi les matériaux, les matières premières, les équipements commerciaux, la propriété intellectuelle, la normalisation, la spécification, certains aspects de l'environnement productif et de la qualité écologique, la protection des droits des consommateurs, les politiques consuméristes et qualitatives, etc. Pour Cochoy et De Terssac (1999), la qualité, en tant que processus, apparaît aujourd'hui comme l'un des maillons centraux de l'entreprise, et ce, pour trois raisons. Premièrement, la qualité permet de cadrer les actions courantes de tous les acteurs impliqués dans la production d'un bien ou d'un service. Deuxièmement, elle veille à réduire l'écart entre «ce qui se fait » et ce qui « doit se faire ». Troisièmement, la qualité contribue à la cohérence des actions des différents niveaux hiérarchiques.

18 Vraisemblablement, la qualité s'inscrit dans la culture de l'entreprise et se construit comme un projet de management en lien avec sa stratégie et ses objectifs; c'est une démarche collaborative qui concerne, en interne, tous les membres de l'entreprise et contribue à la cohésion du groupe vers un objectif commun, et permet, en externe, de consolider la relation avec les clients, les fournisseurs, etc. (DGE et CNS, 2016). Elle permet de renforcer la compétitivité de l'entreprise, améliore son fonctionnement et contribue à la satisfaction et à la fidélisation de ses clients (Ruževičius, 2013). Dès lors, 
l'adoption de la qualité s'avère primordiale car, de la même façon que l'ensemble des outils de gestion, les outils de management de la qualité permettent de formaliser l'action organisée et de rationaliser les processus dans une organisation apportant une logique, une rigueur et une simplification permettant d'atteindre, en cas de succès, une performance optimale de l'entreprise (Bédé et al., 2012).

\section{Méthodologie de la recherche}

19 La revue de littérature montre l'importance, d'une part, de l'accompagnement entrepreneurial, et d'autre part, de la qualité dans la réussite, la pérennisation et la croissance des projets. Toutefois, nous avons remarqué l'absence de recherches qui étudient ces deux facteurs conjointement. C'est pourquoi l'étude restituée dans cet article s'efforce d'étudier la place de l'accompagnement vers la qualité chez les entrepreneurs en phase amont. En effet, dans cette contribution, nous tentons de répondre à la question de savoir : comment les structures d'accompagnement peuventelles inspirer les entrepreneurs en phase de pré-création pour adopter la qualité et la considérer comme un facteur clé de réussite de leurs projets?

Pour ce faire, nous avons choisi de situer notre étude exploratoire à l'intérieur des recherches qualitatives. Selon Hlady-Rispal et Jouison-Laffitte (2015), les questions abordées dans les recherches qualitatives s'attachent à expliquer le "comment » des phénomènes observés. Les auteurs ajoutent que les méthodes qualitatives ont le potentiel d'enrichir les connaissances en entrepreneuriat du fait que les outils mobilisés s'adaptent aux spécificités de ce champ de recherche. Pour leur part, Bühlmann et Tettamanti (2007) avancent que, dans les recherches qualitatives, les chercheurs se préoccupent de mécanismes ou de phénomènes encore peu étudiés à travers des enquêtes de caractère plus exploratoire, avec un fort accent sur la description, une focalisation sur la compréhension des phénomènes et le développement de théories visant à comprendre de la manière la plus complète possible les données recueillies.

21 Pour répondre à notre problématique, nous avons adopté la méthode des cas par entretiens. Cette méthode est appropriée lorsque la question de recherche concerne le " comment» (Yin, 2003). Le terme "cas» désigne le porteur de projet. Notre unité d'analyse est donc l'individu. En raison de la visée purement exploratoire de notre recherche, nous avons adopté une étude multi-sites (plusieurs cas). Ce choix est justifié par deux raisons principales. D'abord, dans une perspective de découverte du phénomène étudié, un de nos objectifs est de rendre compte le plus possible de la diversité et de l'hétérogénéité des expériences des entrepreneurs qui ont trouvé dans les structures d'accompagnement vers la qualité la solution pour assurer la réussite et la survie de leurs projets. Ensuite, la méthode multi-sites est particulièrement adaptée lorsque l'on souhaite, comme c'est notre cas, explorer des aspects peu ou pas étudiés d'un phénomène.

Notre échantillon comprend douze entrepreneurs tunisiens en phase de pré-création (tableau 2). Il s'agit d'un échantillon réduit; ce qui caractérise les études qualitatives dont l'échantillon est plutôt représentatif de l'objet de recherche que d'une population statistique (Hlady-Rispal, 2002). En ce qui concerne le choix des cas, Miles et Huberman (2003) avancent que l'échantillonnage doit être orienté, c'est-à-dire non aléatoire; le 
choix des cas doit se faire pour une finalité de théorisation et non pas pour dégager des résultats de type statistique.

Les données ont été collectées par des entretiens semi-directifs centrés. Il s'agit d'un mode d'entretien dans lequel le chercheur amène le répondant à communiquer des informations nombreuses, détaillées et de qualité sur les sujets liés à la recherche en l'influençant très peu et donc avec des garanties d'absence de biais qui vont dans le sens d'une bonne scientificité (Roussel et Wacheux, 2005). Nous nous sommes adressés à l'ensemble des interviewés avec la même grille de questions, et ce, afin de présenter les différentes perceptions (chacun de sa position, son expérience personnelle et son domaine d'activité) et de l'inspiration que chaque entrepreneur l'a trouvée dans les structures d'accompagnement pour penser à la qualité comme vecteur de réussite entrepreneuriale. La durée moyenne de chaque entretien est de 45 minutes. Le guide d'entretien se structurait autour des questions suivantes:

- Comment trouvez-vous le cadre d'accompagnement à la création d'entreprises ?

- Comment les structures d'accompagnement vous ont-elles aidé ?

- Comment avez-vous pris connaissance des structures d'accompagnement vers la qualité et de leurs services?

- Comment ces structures vous ont-t-elles inspiré pour penser à des choix stratégiques pouvant vous rassurez quant à la réussite et la survie de votre projet?

- Comment jugez-vous l'importance de la qualité pour l'avenir de votre projet?

Les données recueillies ont fait l'objet d'une analyse de contenu. Elles ont été enregistrées, retranscrites dans leur intégralité et sont présentées sous forme de verbatim. Ces verbatim ont subi par la suite un codage thématique en relation avec notre question de recherche (Paillé et Mucchielli, 2012).

Tableau 2 : échantillon de l'enquête

\begin{tabular}{|l|l|}
\hline Entrepreneur & Projet \\
\hline 1 & Sécurité informatique et développement de sites web \\
\hline 2 & Fabrication et vente de produits en inox \\
\hline 3 & Préparation et vente de glace et gâteaux glacés \\
\hline 4 & Menuiserie et vente d'articles en aluminium \\
\hline 5 & Restaurant cuisine asiatique \\
\hline 6 & Fabrication et montage de rideaux automatisés \\
\hline 7 & Vente de produits cosmétiques \\
\hline 8 & Fabrication et installation de panneaux photovoltaïque \\
\hline 9 & Production et vente de plantes de décoration \\
\hline 10 & Fabrication et installation d'enseignes lumineuses \\
\hline 11 & Fabrication et installation de mécanismes d'alarmes \\
\hline
\end{tabular}


\begin{tabular}{l|l}
12 & Centre privé de cours particuliers
\end{tabular}

\section{Résultats et analyse}

\subsection{L'accompagnement et la création d'entreprises}

socioéconomique, mais leur survie demeure beaucoup plus importante. Cette survie n'est pas liée à l'entrepreneur uniquement ou à son entreprise, elle dépend de plusieurs facteurs et conditions en relation avec son entourage, l'environnement général de l'entreprise créée, les divers mécanismes, programmes et structures d'accompagnement, etc. (Ben Ali, 2020 ; Kouada et al., 2018; Wolff et Cuénoud, 2017 ; Theodoraki et Messeghem, 2016).

"La réussite des entreprises nouvellement créées donne un sens à leur création. Il ne suffit pas de lancer de nouveaux projets, mais il est plus important d'assurer leur survie. Personne ne peut nier que cette survie est la résultante d'une combinaison de facteurs liés au porteur de projet (son profil, sa situation, son entourage, etc.), à son projet (déroulement, gestion, etc.), à l'environnement avec toutes ses dimensions, etc. Un des facteurs clés de succès des nouveaux projets est l'accompagnement par les différentes structures instaurées. Sans accompagnement, les entreprises nouvelles, généralement très fragile aux aléas de l'environnement, risquent de fermer leurs portes très rapidement ». (Entrepreneur 7)

29 À propos de l'importance de l'accompagnement dans la réussite entrepreneuriale, Tounès (2003), en parlant du rôle joué par l'État dans le développement des activités entrepreneuriales, souligne que celles-ci ne peuvent voir le jour et survivre que par la mise en place de certains mécanismes institutionnels et fiscaux. Dans cette optique, les politiques économiques des pays qui ont choisi de miser sur l'entrepreneuriat pour assurer leur développement doivent être particulièrement importantes afin d'assurer la réussite de telles politiques.

"Je pense que les structures d'accompagnement ont un rôle important dans le lancement des nouveaux projets. Ce rôle est confirmé quand les entreprises nouvellement créés trouvent l'assistance et le soutien après leur démarrage. La survie de plusieurs entreprises est due essentiellement aux apports des différentes structures ». (Entrepreneur 12)

31 En plus de l'entrepreneur et de son processus entrepreneurial, le contexte général influence la création d'entreprises et façonnent leur impact socioéconomique (Abdennadher, 2012). Pour Botzung (1996, p.136), «l'appui aux petites entreprises 
constitue un champ d'opération et d'investigation au croisement de l'entreprise, de l'entrepreneur et du trinôme État/bailleur/opérateur... L'entrepreneur apporte son savoir-faire, son itinéraire entrepreneurial et ses références sociales intériorisées... Les dispositifs d'appui visent à lever les hypothèques pesant sur l'environnement de l'entreprise, conseillent et élaborent des formations à l'attention des entrepreneurs... ». Cuzin et Fayolle (2006, p. 92) avancent que «l'accompagnement est souvent présenté comme l'un des facteurs de réussite des projets de création, reprise ou développement d'entreprise. Les pays dans lesquels la densité entrepreneuriale est la plus forte sont aussi ceux où l'accompagnement précoce est le plus développé ".

\subsection{L'apport des structures d'accompagnement aux entrepreneurs}

La création d'entreprises et leur survie est la résultante d'une combinaison d'efforts, de conditions et de facteurs. En Tunisie, dans une démarche de développement économique, de nombreuses structures et mécanismes d'accompagnement à la création d'entreprises ont été instaurées dans le but d'offrir aux entrepreneurs des services et des produits pour leur faciliter la création de leurs entreprises et leur assurer la survie et le développement de leurs projets (Ben Ali, 2020 ; Haddad et Melliti, 2018 ; Ben Taher Gheryaani et Boujelbène, 2017). Les différentes structures mises en place depuis quelques années ne cessent d'offrir des services pour les entrepreneurs qui se préparent pour lancer leurs projets et pour ceux qui se sont déjà installés car même si l'entrepreneur est l'auteur principal de son projet, il ne peut pas toutefois réussir seul son parcours (Ben Ali, 2020 ; Pluchart, 2013 ; Barès et Muller, 2007).

"Auparavant, je croyais que la création d'une entreprise est l'affaire de l'entrepreneur dans la mesure où il doit se casser la tête pour lancer son projet. Toutefois, quand j'ai commencé à me renseigner sur les différentes procédures, j'étais surpris de la multitude de structures existantes qui interviennent pour faciliter le processus de création ». (Entrepreneur 4)

34 L'apport des différentes structures d'accompagnement à la création d'entreprises est d'une grande importance du fait qu'elles soutiennent les entrepreneurs en leur facilitant la démarche entrepreneuriale par des services et des produits au moment du montage de leurs projets et même après pour leur aider à se développer (Kouada et al., 2018; Wolff et Cuénoud, 2017; Theodoraki et Messeghem, 2016; Verzat et Toutain, 2015 ; Messeghem et al., 2014 ; Pluchart, 2013 ; Grossetti et Barthe, 2008).

"Dans sa quête de création, l'entrepreneur a besoin de quelqu'un qui l'écoute, l'assiste, l'aide, le soutient, l'informe, l'oriente, etc. Et à ce niveau les différentes structures que j'ai visitées sont extrêmement utiles ». (Entrepreneur 6)

"À travers des cellules d'écoute et l'organisation de certaines manifestations, ces structures mettent à la disposition des entrepreneurs une documentation riche et un ensemble d'actions leur permettant d'acquérir une certaine visibilité sur les procédures et les formalités de création, les aides dont ils pourront bénéficier, etc. ». (Entrepreneur 9)

En effet, il existe différents niveaux d'intervention à travers lesquels les structures d'accompagnement sont en mesure d'aider les entrepreneurs depuis la phase de précréation jusqu'à la création proprement dite et même après le démarrage pour leur assurer la continuité de leurs projets. Hentic-Giliberto et Berger-Douce (2017) montrent que l'accompagnement dès la phase amont permet aux entrepreneurs de mieux appréhender la pertinence de leur projet et l'intérêt de sa mise en œuvre effective. Ainsi, tout entrepreneur a besoin d'être écouté, informé, formé, conseillé, orienté, 
assisté et suivi durant la phase de pré-création, au moment de la création et après le démarrage de l'activité; ce qui constitue la principale mission des structures d'accompagnement.

"L'appui et l'accompagnement touchent plusieurs volets. D'abord, le volet financier qui demeure déterminant surtout que la plupart des projets lancés sont sous formes de petites entreprises avec des créateurs qui souffrent d'un manque de fonds. Après, viennent l'assistance et le conseil en matière de procédures administratives, juridiques, fiscales, etc. Ensuite, il y a le volet lié à tout ce qui se rapporte au développement des compétences techniques et managériales". (Entrepreneure 1)

L'accompagnement et l'assistance des différentes structures touchent plusieurs aspects de la création d'entreprises. Les plus importants restent l'accès au financement, le soutien technique et logistique, l'information liée aux différentes procédures administratives, juridiques, fiscales, etc., et les formations permettant aux entrepreneurs d'acquérir et développer des compétences techniques et managériales utiles au lancement des projets, mais aussi et surtout pour leur assurer la pérennisation de leurs entreprises durant les premières années de démarrage (Haddad et Melliti, 2018; Degeorge, 2017 ; Mhamed Hichri et al., 2017 ; Theodoraki et Messeghem, 2016; Fayolle et al., 2010).

\subsection{L'accompagnement et la qualité au service de l'entrepreneur}

\subsubsection{L'accompagnement comme source d'inspiration en matière de qualité}

Afin qu'il soit efficace, l'accompagnement des entrepreneurs doit s'appliquer tout au long du processus entrepreneurial ; de l'intention à la création et durant les premières années de l'existence du projet. Pour Deschamps et al. (2010), l'accompagnement ne peut être performant que s'il s'inscrit dans la durée. En effet, la pérennisation de l'entreprise nouvellement créée est tributaire d'un accompagnement qui s'effectue dans le temps étant donné leur fragilité face à certains problèmes qui peuvent menacer leur survie (Sammut, 2003). À ce niveau, le rôle de l'accompagnement est d'accorder aux entrepreneurs les compétences nécessaires pour préserver leurs projets.

"L'intention de lancer mon projet raisonnait toujours dans ma tête, mais ce qui m'inquiétait le plus c'est comment faire pour assurer la survie de mon entreprise. Face à la concurrence qui se joue depuis des années à la fois à l'échelle nationale qu'internationale et devant les attentes des clients et des consommateurs qui sont devenus avertis et de plus en plus exigeants, je me demandais comment se comporter. La solution m'est venue en consultant un document présentant l'INNORPI ${ }^{1}$. (Entrepreneur 6)

42 Étant donné leurs retombées positives, la pérennisation des entreprises nouvellement créées est importante aussi bien pour les entrepreneurs que pour toutes les parties prenantes. Cette pérennisation est tributaire du bon déroulement de la phase de précréation comme le montrent Bornard et al. (2019) qui soulignent que l'accompagnement entrepreneurial lors des phases amont du processus de création demeure pertinent. En effet, la réussite du projet et sa pérennisation nécessite l'effort de tout un système en partant de l'entrepreneur lui-même (profil, caractéristiques, motivations, etc.) (Mahamat et al., 2020; Benchikh et al., 2019; Glidja, 2019), son entourage (réseaux de relations, famille, etc.) (Randriamanalina, 2020 ; Ojong et Simba, 2019 ; Abdennadher et al., 2015 ; Grossetti et Barthe, 2008) pour arriver à l'ensemble des 
structures d'accompagnement (Ben Ali, 2020 ; Bornard et al., 2019 ; Haddad et Melliti, 2018 ; Hentic-Giliberto et Berger-Douce, 2017 ; Pluchart, 2014 ; Deschamps et al., 2010 ; Cuzin et Fayolle, 2006). L'apport de ces dernières est considérable du fait qu'elles contribuent à un large éventail de compétences indispensables non seulement pour la survie de l'entreprise en démarrage, mais aussi et surtout pour le développement de son avantage compétitif (Fayolle et al., 2010).

43 «La qualité, parfois coûteuse, reste déterminante dans le développement de l'entreprise. Elle permet d'innover constamment, d'améliore la communication interne et externe, de maintenir et enrichir les compétences de tous les intervenants, d'améliorer la qualité des produits et de rester compétitif afin d'être plus efficace et plus performant ce qui permet de préserver les clients et gagner d'autres. Un ami, déjà installé pour son propre compte et qui adopte la qualité, m'a conseillé de s'adresser à l'INNORPI ». (Entrepreneur 11)

Ainsi, un des facteurs qui permet à la jeune entreprise d'assurer sa croissance et sa continuité est la possession d'un avantage concurrentiel qui peut se réaliser à travers l'adoption d'une démarche basée sur la qualité (Ruževičius, 2013; Bédé et al., 2012; Cochoy et De Terssac, 1999). La concurrence qui s'exerce sur les marchés opère un processus de sélection; seules les entreprises les plus performantes survivent (Petrella et Richez-Battesti, 2010). À ce niveau, l'INNORPI, la structure d'accompagnement à la création d'entreprises la plus citée par les entrepreneurs de notre échantillon, joue un rôle important dans la diffusion de la culture de la qualité. Cette structure informe, oriente, forme et assiste les entrepreneurs dans l'adoption d'une démarche qualité dès la phase de pré-création.

45 «Seulement les entrepreneurs qui ont pensé sérieusement à adopter la qualité peuvent garantir la réussite de leur parcours. Quant à moi, c'est grâce à l'INNORPI que j'ai eu l'idée d'opter pour la qualité dans l'avenir ». (Entrepreneur 10)

46 Désormais, la qualité est de plus en plus importante dans tous les domaines, toutes les activités, toutes les organisations et dans toutes les sphères de la vie d'individu et de la société (Ruževičius, 2013). Les entreprises qui souhaitent survivre et se développer dans un environnement fortement concurrentiel sont appelées à investir dans la qualité. Selon Cochoy et De Terssac (1999), l'ultime justification de la qualité est celle du marché, de la concurrence et du client.

47 "Le souci majeur de tout entrepreneur en phase de pré-création est de trouver une réponse à la question suivante : comment faire pour réussir mon projet et assurer sa continuité? J'ai trouvé la réponse à cette question lors de ma participation à une journée d'information destinées aux entrepreneurs. Et là, j'ai découvert l'INNORPI. L'organisme dont la mission est d'entreprendre toute action concernant la normalisation, la qualité des produits et services, la protection de la propriété industrielle, etc. En effet, jouer la carte de la qualité est devenue une des meilleures solutions pour parer la concurrence, répondre aux besoins des clients et des consommateurs, gagner en part de marché, etc. En quelques sortes, garantir la survie de l'entreprise». (Entrepreneur 11)

48 Désormais, la qualité apparaît comme l'un des maillons décisifs de l'action organisationnelle qui se présente comme une chaîne de décisions visant à orienter le comportement des différents acteurs qui contribuent à la production d'un bien ou d'un service (Cochoy et De Terssac, 1999). Certes, les entreprises qui veulent rester dans le marché doivent faire beaucoup plus attention à la qualité étant donné que les revenus et les connaissances des consommateurs augmentent et qu'ils commencent à chercher de la qualité (Ruževičius, 2013). Dans cette optique, l'apport des structures 
d'accompagnement est d'une grande utilité pour les entrepreneurs qui se préparent à lancer leurs projets et qui sont parfois mal informés sur les politiques et les stratégies existantes pouvant leur assurer la réussite de leurs projets. C'est le cas des entrepreneurs de notre échantillon qui ont trouvé dans l'INNORPI l'accompagnement nécessaire qui les a inspirés pour penser à la qualité et la considérer comme la pièce maîtresse de la réussite et la survie de leurs entreprises. En 1982, dans une logique de protection de la propriété industrielle et pour promouvoir la culture de la qualité, le gouvernement tunisien a fondé l'INNORPI (Mabrouki, 2017 ; Ben Henda, 2011). En tant que structure d'accompagnement à la création et au développement d'entreprises, l'INNORPI est chargée d'entreprendre toute action concernant la normalisation, la qualité des produits et services, la métrologie, la protection de la propriété industrielle et la tenue du registre central du commerce ${ }^{2}$.

\subsubsection{L'apport de I'INNORPI pour les entrepreneurs en matière de qualité} tendance en matière de qualité. En fouillant les différents documents surtout ceux qui concernent la qualité, j'ai compris que la donne a bien changé et que pour pouvoir réussir, il faut s'orienter vers la qualité». (Entrepreneur 5)

51 Pour promouvoir la qualité de leurs produits, les entreprises peuvent faire recours à la certification qui se présente comme un outil indispensable dans la démarche qualité (Agnès, 1996). Désormais, ce sont les clients qui font vivre l'entreprise en achetant ou non ses produits et services (Giordano, 2006). Certes, le client est le seul juge de la qualité, il ne tolère plus « le mauvais », « le bon » est la norme ; la qualité est devenue indispensable (Guilhon et Weill, 1996).

"Une des missions de l'INNORPI est la certification. Celle-ci consiste à certifier la conformité des produits aux exigences d'une norme. La certification s'avère un élément clé du positionnement d'un produit par rapport à celui de la concurrence. Toujours en relation avec la concurrence, la certification permet, entre autres, de consolider le processus de fabrication, de donner une meilleure connaissance des qualités du produit, l'optimisation de la gestion de l'entreprise, etc. ». (Entrepreneur 9)

53 Delvosalle (2002) avance qu'il est difficile de parler de la qualité sans se voir immédiatement interrogé sur la certification des systèmes qualité, les normes ISO 9000, le manuel qualité, etc.

54 "En matière de certification, l'INNORPI a pour mission de certifier les produis et les systèmes de management. Les documents disponibles montrent l'importance de cette certification dans la possession d'un avantage concurrentiel. Certifier la qualité d'un produit, c'est attester sa conformité aux exigences d'une norme ce qui permet de garantir d'offrir un produit concurrentiel. En ce qui concerne la certification des systèmes, il est à noter l'importance de l'adoption d'un système de management de la qualité car celui-ci peut aider l'entreprise à 
réaliser des bénéfices et, d'une façon générale, améliorer ses performances globales et d'assurer sa pérennité». (Entrepreneur 7)

En ce qui concerne les critères de qualité des produits, ceux-ci sont fixés par la normalisation tout en spécifiant les performances à atteindre en matière de fiabilité, de durabilité, d'information, de sécurité, d'environnement ou encore d'hygiène (Agnès, 1996). La normalisation se définit comme une activité d'intérêt général dont l'objet est de fournir des documents de référence portant sur des règles, des caractéristiques, des recommandations ou des exemples de bonnes pratiques, relatives à des produits, des services, des méthodes, des processus ou des organisations, visant à encourager le développement économique et l'innovation tout en prenant en compte des objectifs de développement durable (Evrard, 2014).

56 "L'application des normes n'est pas obligatoire, mais quand tu te renseignes sur l'ensemble des normes, leurs intérêts et leurs avantages, tu te trouves dans l'obligation d'adopter une telle démarche. En se référant à l'INNORPI, la qualité peut être assurée par le recours à la normalisation puisqu'elle permet de répondre aux attentes de l'ensemble des acteurs socioéconomiques et, notamment, de donner confiance que ce soit pour les produits ou les services ce qui peut permettre à l'entreprise de favoriser sa compétitivité et, en quelque sorte, d'assurer sa survie ». (Entrepreneur 2)

57 La normalisation, dont l'un de ses objets est de garantir la qualité et la fiabilité des produits ainsi que la sécurité des usagers, a été auparavant à l'origine de l'apparition d'une multitude de signes de qualité (les normes, les marques, les labels, etc.) constituant autant de moyens d'appréciation de la qualité des biens et services offerts (Agnès, 1996). Comme le montrent les différents documents publiés par l'INNORPI, une norme couvre un ensemble de spécifications techniques qui doivent être respectées visant à garantir le plus haut niveau de qualité des biens, des services et des activités (Gómez et Pinto, 2001).

58 "Les informations diffusées par l'INNORPI m'ont été d'une grande importance pour construire une idée sur les différentes normes dans tous les secteurs, mais aussi et surtout sur celles qui concernent mon secteur d'activité. La production en conformité de ces normes permet de garantir des produits et des services sûrs, fiables et de bonne qualité ». (Entrepreneur 8)

59 Ainsi, la qualité apparaît comme un moyen par lequel les entrepreneurs qui se préparent à lancer leurs entreprises peuvent réaliser un avantage compétitif et assurer pour eux une sorte de garantie leur permettant de joindre la croissance à la pérennité. Grâce à la qualité, le client est rassuré parce que ce qu'il achète est conforme au produit qu'il attend (Cochoy et De Terssac, 1999). En contrepartie, une mauvaise qualité place l'entreprise en position de désavantage concurrentiel car si les clients perçoivent que la qualité n'est pas satisfaisante, ils ne tarderont pas à aller voir ailleurs (Giordano, 2006; Guilhon et Weill, 1996). Au cœur de la démarche qualité, la satisfaction et la fidélisation des clients constituent l'enjeu majeur pour toute entreprise cherchant à préserver et améliorer sa compétitivité (DGE et CNS, 2016).

60 «La qualité contribue à la structuration de l'organisation et pose les ingrédients de la pérennisation de l'entreprise. Elle permet de répondre aux attentes des clients et de réaliser une différenciation par rapport à la concurrence. C'est une démarche d'amélioration continue et une preuve de professionnalisme ». (Entrepreneur 4)

61 La normalisation est une activité d'intérêt général qui permet le développement économique durable et qui constitue un outil stratégique pour les différents acteurs 
économiques leur permettant d'obtenir des avantages concurrentiels (Evrard, 2014). Les entrepreneurs trouvent donc dans les normes un outil efficace pour travailler, produire et vendre des produits de qualité permettant d'assurer la viabilité de leurs projets. Elles ont un impact positif du fait qu'elles peuvent maintenir et améliorer la qualité, apporter des informations et assurer l'interopérabilité et la compatibilité, augmentant de ce fait la sécurité et la valeur pour les consommateurs (Evrard, 2014). À ce niveau, le rôle des structures d'accompagnement s'avère d'une grande utilité. Dans notre cas, l'INNORPI permet de familiariser les entrepreneurs aux normes et leur présente les avantages qui résultent de leur adoption. La normalisation est un facteur important de compétitivité car elle permet de maitriser les coûts, d'assurer la bonne gestion, de renforcer la capacité concurrentielle, etc. ce qui permet d'apporter tant de bénéfices pour l'entrepreneur et son entreprise.

"L'INNORPI est une structure qui m'a beaucoup marqué. Elle m'a permis d'avoir une idée sur les différentes normes existantes, leur rôle, leur importance, leur intérêt, etc. Toutes les données qui m'ont été fournies m'ont inspiré; j'ai décidé de jouer la carte de la qualité dans sa globalité. Je compte gérer mon entreprise tout en respectant et appliquant les normes afin de garantir le maximum de qualité pour assurer le bon fonctionnement de mon projet et gagner la confiance de mes futures clients ». (Entrepreneur 3)

La survie de l'entreprise est liée principalement à sa capacité à réaliser des bénéfices qui seront à l'origine de la performance financière. Cette performance est basée sur le client dont la satisfaction est l'objectif central de la qualité et qui dépend non seulement de la capacité de l'entreprise à offrir un produit de qualité, mais surtout de sa capacité à générer la qualité dans tous ses processus stratégiques (Guilhon et Weill, 1996) car, selon Cochoy et De Terssac (1999), ce qui vaut pour l'organisation dans son ensemble vaut sûrement pour l'ensemble de ses compartiments. Ainsi, le recours à la qualité ne concerne pas uniquement les produits et les services de l'entreprise, mais les entrepreneurs seront en mesure de l'adopter dans sa globalité pour toucher la gestion quotidienne, le management stratégique, etc.

\section{Conclusion}

Pour réussir leurs projets et assurer la survie de leurs entreprises, les entrepreneurs qui souhaitent s'installer pour leur propre compte doivent adopter des stratégies leur permettant de préserver un avantage concurrentiel, satisfaire les clients et gagner des parts de marché. Certes, la réussite des projets ne concerne pas uniquement les entrepreneurs; c'est le résultat d'un effort commun là où les structures d'accompagnement jouent un rôle de premier ordre. Les résultats de notre recherche montrent que les entrepreneurs en phase de pré-création comptent énormément sur l'apport des structures d'accompagnement pour oser l'aventure entrepreneuriale, assurer un meilleur démarrage et maximiser les chances de réussite de leurs projets. Cet accompagnement couvre une assistance à la fois matérielle et immatérielle; financière, administrative, psychologique, technique, managériale, etc. (Ben Ali, 2020 ; Belhaj et al., 2018; Haddad et Melliti, 2018; Degeorge, 2017 ; Mhamed Hichri et al., 2017 ; Theodoraki et Messeghem, 2016 ; Pluchart, 2013 ; Fayolle et al., 2010). Désormais, l'accompagnement des entrepreneurs est considéré comme un facteur essentiel pour que ces derniers puissent parcourir avec succès leur processus entrepreneurial. Cet accompagnement ne s'effectue pas à un moment précis, mais doit se faire avant, au 
moment et après la création pour garantir le bon démarrage et la survie de l'entreprise (Ben Ali, 2020 ; Messeghem et al., 2014 ; Abdennadher, 2012 ; Grossetti et Barthe, 2008).

Soucieux de leur avenir et de celui de leurs projets, et durant cette phase de précréation, les entrepreneurs, de plus en plus confrontés à bon nombre de défis socioéconomiques menaçant l'achèvement du processus de création, pensent aux facteurs qui peuvent contribuer au succès de leur parcours entrepreneurial et surtout à ceux qui sont susceptibles d'assurer la survie de leurs entreprises. À ce niveau, les entrepreneurs de notre échantillon se sont mis d'accord sur l'importance de l'INNORPI, comme structure d'accompagnement, qui vient pour éclairer l'importance de l'adoption d'une démarche qualité. Grâce à cette structure, les entrepreneurs ont pu construire une idée sur la qualité et connaître son importance pour l'avenir de leurs projets. À travers ses actions qui concernent la normalisation, la certification, la protection de la propriété industrielle, la formation, la communication et l'information, l'INNORPI a pu inspirer les entrepreneurs de la nécessité de l'adoption d'une démarche qualité. Grâce à cette structure, les entrepreneurs ont pris conscience que la qualité et la survie de l'entreprise sont intimement liées.

En effet, les entrepreneurs en phase de pré-création ont trouvé la formule qui peut leur "garantir» la pérennisation de leurs projets auprès d'une structure d'accompagnement vers la qualité ; c'est sur la qualité qu'il faut miser, et c'est par l'accompagnement des structures ad hoc que ces entrepreneurs peuvent surmonter les obstacles et pourront ainsi satisfaire et fidéliser leurs clients, posséder un avantage compétitif et assurer la performance de leurs entreprises. À ce propos, Grossetti et Barthe (2008) avancent que les entreprises accompagnées sont plus pérennes et plus performantes que les entreprises non accompagnées. Bédé et al. (2012) ajoutent que l'adoption d'une démarche de la qualité contribue fortement au succès de l'entreprise et à la réalisation d'une performance optimale. Ainsi, l'accompagnement et la qualité apparaissent comme deux facteurs qui jouent un rôle déterminant dans le lancement, la réussite et la survie des entreprises; il serait donc très utile pour les entrepreneurs d'être accompagnés dans l'instauration de la qualité. En fait, savoir qu'ils pourront être accompagnés vers la qualité constitue pour les entrepreneurs en phase de pré-création un réconfort tout particulier pour aller de l'avant dans leur aventure entrepreneuriale.

\section{Les apports de l'étude}

67 Notre étude présente deux principales contributions théoriques. Tout d'abord, elle contribue à mieux comprendre l'importance de l'accompagnement durant l'aventure entrepreneuriale et l'apport des structures pour appuyer, soutenir et assister l'entrepreneur afin de réussir son projet. Ensuite, elle met en évidence le rôle de la qualité dans la croissance et la pérennisation des entreprises.

Empiriquement, notre enquête confirme l'intérêt de l'entrepreneur de s'adjoindre aux structures d'accompagnement vers la qualité dès la phase de pré-création pour accéder à l'ensemble des services et ressources disponibles. L'étude montre l'intérêt d'une approche globaliste de l'accompagnement et de la qualité. Ces deux facteurs, quand ils sont associés, offrent aux entrepreneurs en phase de pré-création un sentiment de réconfort quant à la réussite de leurs parcours. Par conséquent, un accompagnement dès les premières étapes du projet permet d'inspirer les entrepreneurs à opter pour la qualité afin de maximiser les chances de réussites de leurs entreprises. 


\section{Les limites de l'étude} donc peu varié. En plus, une limite en relation avec la recherche exploratoire qui fait appel à la mémoire des entrepreneurs ce qui renvoie à trois biais. D'abord, le biais de mémoire du fait qu'en répondant, l'individu attache de l'importance aux seuls éléments dont il se souvient. Ensuite, le biais de désirabilité sociale dans la mesure où l'interrogé dresse un tableau de ses pratiques en accord avec l'image qu'il veut refléter. Enfin, le biais de rationalisation puisque l'entrepreneur a tendance à rendre cohérents les phénomènes qu'il évoque. La triangulation de nos données avec d'autres techniques de collecte de données permettra de dépasser ces limites. L'interrogation d'autres entrepreneurs et des responsables des structures d'accompagnement vers la qualité pourra aussi limiter ces biais. Ceci peut, en effet, faire l'objet de prolongements futurs de notre recherche.

Conceptuellement, notre étude présente une limite relative au choix des entrepreneurs en phase de pré-création. Les autres phases du projet peuvent être intégrées dans l'analyse; un tel enrichissement est intéressant car il permet de mieux saisir l'importance de l'accompagnement vers la qualité pour les entrepreneurs.

\section{BIBLIOGRAPHIE}

Abdennadher S. (2012), Les facteurs contextuels dans l'acte entrepreneurial : cas de la Tunisie, Thèse de Doctorat en Sciences de Gestion, FSEGS, Université de Sfax, Tunisie.

Abdennadher S. et al., (2015), « Influence de la famille et du réseau relationnel sur l'acte entrepreneurial : cas des entrepreneurs tunisiens » Question(s) de management, 2 (10), pp. 11-21.

Agnès G. (1996), « Normalisation, certification : quelques éléments de définition » Revue d'économie industrielle, 75 (1), pp. 45-60.

Aouni Z. et B. Surlemont, (2007), « Le processus d'acquisition des compétences entrepreneuriales : une approche cognitive " $5^{\text {ème }}$ Congrès de l'AEI, Sherbrooke, Octobre, 4-5.

Barès F. et R. Muller, (2007), « Appui à la création d'entreprise : du narcissisme au partage de ressources. L'exemple d'un réseau d'aide à la création d'entreprise » Revue Internationale de Psychosociologie, 13 (31), pp. 125-146. 
Bédé D. et al., (2012), « L'appropriation d'un outil de gestion de la qualité à travers le prisme de la légitimité : le cas d'un Institut d'Administration des Entreprises » Management \& Avenir, 4 (54), pp. 83-106.

Belhaj M. et al., (2018), Rapport sur la Cartographie des dispositifs existants dans les villes de Sfax et Carthage - Tunisie, Université de Sfax.

Ben Ali T. (2020), « Le management entrepreneurial au service du développement régional » Revue de Recherches en Economie et en Management Africain, 8 (8), pp. 76-95.

Ben Henda M. (2011), « Normes e-Learning pour une interopérabilité universitaire en Tunisie » $1^{\text {st }}$ International Symposium ISKO-Maghreb, Hammamet, Mai, 13-14.

Ben Taher Gheryaani S. et Y. Boujelbène, (2017), « Survie des jeunes entreprises : Impact de l'accompagnement entrepreneurial, des caractéristiques des entrepreneurs et de leurs projets » $12^{2 \grave{m} e}$ Colloque scientifique en finance, Djerba, Décembre, 19-20.

Benchikh M. et al., (2019), « Le rôle du profil de l'entrepreneur dans la réussite des PME Algériennes » Revue Algérienne d'économie de gestion, 12 (2), pp. 82-110.

Bornard F. et al., (2019), « Accompagnement entrepreneurial : le point de bascule » Entreprendre \& Innover, 40 (1), pp. 77-93.

Botzung M. (1996), « Dispositifs d'appui et financement de la petite entreprise » Revue TiersMonde, 37 (145), pp. 135-151.

Bounouh A. (2017), « Développer la culture d'entreprise chez les jeunes par la formation? Eléments de diagnostic et enjeux en Tunisie » Forum international de l'économie sociale et solidaire, Marrakech, Mai, 22-24.

Bühlmann F. et M. Tettamanti, (2007), « Le statut de l'approche qualitative dans des projets de recherche interdisciplinaires » Recherches Qualitatives, Hors Série (3), pp. 191-213.

Chihi Y. (2005), « Entrepreneuriat et migration : le cas de sud-ouest tunisien » $2^{\text {ème }}$ Rencontre jeunes et sociétés en Europe et autour de la Méditerranée, Octobre, 24-25.

Cochoy F. et G. De Terssac, (1999), « Les enjeux organisationnels de la qualité : une mise en perspective » Sciences de la Société, Presses universitaires du Midi, 46, pp. 3-18.

Cuzin R. et A. Fayolle, (2006), « Quel appui à la création d'entreprises ? »'Expansion Management Review, 1 (120), pp. 92-97.

Da Silva N. (2018), « L'industrialisation de la médecine libérale : une approche par l'Économie des conventions » Management \& Avenir Santé, 1 (3), pp. 13-30.

Degeorge J-M. (2017), « De la diversité du processus d'accompagnement entrepreneurial vers une meilleure complémentarité » Revue de l'Entrepreneuriat, 16 (2), pp. 7-15.

Delvosalle C. (2002), « La qualité : des concepts à la pratique » Pyramides, 5, pp. 137-154.

Deschamps B-P. et al., (2010), « Accompagner le repreneur d'entreprise : conduire, escorter mais aussi guider » Gestion 2000, 27 (3), pp. 77-88.

DGE et CNS, (2016), La qualité, levier de performance des PME de services, Guide pratique.

El Amrani J. et al. (2016), « Étude exploratoire de l'écosystème d'accompagnement à

l'entrepreneuriat social au Maroc »13 ${ }^{\text {ème }}$ CIFEPME, Trois Rivières, Octobre, 26-28. 
El Kahri L. et al., (2014), « La certification qualité et la performance des organisations : Quelles convergences ? Étude de cas au sein de la Nouvelle Société AMADIR dans le grand Agadir » International Journal of Innovation and Scientific Research, 11 (2), pp. 598-610.

Evrard L. (2014), Politique nationale de normalisation et stratégie pour la compétitivité de notre économie, Rapport Ministériel, France.

Eymard-Duvernay F. (1995), « La négociation de la qualité » In Nicolas F. et Valceschini E. (eds.), Agro-alimentaire : une économie de la qualité, Paris, INRA, Economica, pp. 39-48.

Eymard-Duvernay F. (1989), «Conventions de qualité et formes de coordination » Revue Économique, 40 (2), pp. 329-359.

Fatien P. (2008), « Des ambiguïtés des maux/mots du coaching » Nouvelle revue de psychosociologie, 2 (6), pp. 193-211.

Fayolle A. (2004), « Compréhension mutuelle entre les créateurs d'entreprise et les accompagnateurs : une recherche exploratoire sur les différences de perception » Management International, 8 (2), pp. 1-14.

Fayolle A. et al., (2010), « Efficacité des pépinières dans la création d'entreprise innovante : cas de la Tunisie » Innovations, 3 (33), pp. 157-179.

Fortin P-A. (2002), La culture entrepreneuriale : un antidote à la pauvreté, Québec, Les Éditions de la Fondation de l'Entrepreneurship.

Gagnon É. et al., (2011), « Ce qu'accompagner veut dire » Reflets, 17 (1), pp. 90-111.

Giordano J-L. (2006), L'approche qualité perçue, Paris, Éditions d'Organisation.

Glidja J. (2019), « Les déterminants du succès de l'entrepreneuriat féminin au Bénin, le rôle modérateur de l'appui institutionnel : cas de la WBPC » Gestion 2000, 36 (2), pp. 39-59.

Gómez C. et M. Pinto, (2001), « La normalisation au service du traducteur » Meta, 46 (3), pp. 564579.

Grossetti M. et J-F. Barthe, (2008), « Dynamique des réseaux interpersonnels et des organisations dans les créations d'entreprises » Revue Française de Sociologie, 49 (3), pp. 585-612.

Guilhon A. et M. Weill, (1996), « Démarche qualité : de la stratégie d'adaptation aux processus stratégiques de changements dans les PME »V Vème Conférence de l'AIMS, Lille, Mai, 13-15.

Haddad S. et N. Melliti, (2018), « Rôle des structures d'accompagnement dans la création des entreprises innovantes en Tunisie. Cas des pépinières de la région du Sahel Tunisien » Marché et Organisations, 33 (3), pp. 79-104.

Hentic-Giliberto M. et S. Berger-Douce, (2017), « Rôle de l'accompagnement amont sur la poursuite du projet d'affaires : résultats d'une enquête empirique » Revue de l'Entrepreneuriat, 16 (2), pp. 27-57.

Hernandez É-M. (1999), Le processus entrepreneurial : vers un modèle stratégique d'entrepreneuriat, Paris-Montréal, L'Harmattan.

Hlady-Rispal M. (2002), La méthode des cas : Application à la recherche en gestion, De Boeck Supérieur. Hlady-Rispal M. et E. Jouison-Laffitte, (2015), « La contribution des méthodes qualitatives au développement du champ de l'entrepreneuriat » Revue de l'Entrepreneuriat, 14 (1), pp. 15-40. Hoang H. et B. Atoncic, (2003), « Network-based research entrepreneurship: a critical review » Journal of business venturing, 18 (2), pp. 165-187. 
Issor Z. (2017), « La performance de l'entreprise : un concept complexe aux multiples dimensions » Projectics / Proyéctica / Projectique, 17 (2), pp. 93-103.

Julien P-A. (2003), « Innovation et entrepreneuriat » In Mustar P. et H. Penan, (Dir.), Encyclopédie de l'innovation, Economica, pp. 167-178.

Kouada S. et al., (2018), « L'hypercroissance des start-up n'est pas un long fleuve tranquille : rôle et place des structures d'accompagnement? » $14^{\text {ème }}$ CIFEPME, Toulouse, Octobre, 23-26.

Lechner et al., (2006), « Firm networks and firm development: the role of the relation mix » Journal of business venturing, 21 (4), pp. 514-540.

Mabrouki M. (2017), Protection par la propriété industrielle et innovation : une analyse dans le cadre de l'économie tunisienne, MPRA Paper, University Library of Munich, Germany.

Mahamat A-A. et al., (2020), « Profil psychologique des propriétaires-dirigeants et innovation entrepreneuriale des PME au Cameroun » Global Journal of Management and Business Research : A Administration and Management, 20 (7), pp. 20-30.

Mas-Verdú, F. et al. (2015), « Firm survival: The role of incubators and business characteristics » Journal of Business Research, 68 (4), pp. 793-796.

Mathot P. (2010), Accompagner l'entrepreneuriat, un impératif pour la croissance, Rapport de mission, France, Octobre.

Mhamed Hichri S-M. et al., (2017), « Rôle des pépinières, caractéristiques du projet entrepreneurial et croissance des start-ups TIC : le cas d'un pays en développement » Revue de l'Entrepreneuriat, 16 (2), pp. 59-90.

Messeghem K. et al., (2014), Panorama des structures d'accompagnement en termes de management et de performance, Labex Entreprendre, Université de Montpellier.

Messeghem K. et al., (2010), «Construction d'un outil stratégique d'évaluation de l'accompagnement à la création d'entreprise : Apport du tableau de bord prospectif » Gestion 2000, 27 (2), pp. 95-112.

Miles M-B. et M-A. Huberman, (2003), Analyse des données qualitatives, Bruxelles, De Boeck, $2^{\text {ème }}$ Édition.

Ojong N. et A. Simba, (2019), « Favoriser le capital social structurel et relationnel des microentrepreneurs par la microfinance » Journal of Small Business \& Entrepreneurship, 31 (1), pp. 1-20.

Paillé P. et A. Mucchielli, (2012), L'analyse qualitative en sciences humaines et sociales, Paris, Armand Colin, $3^{\text {ème }}$ Édition.

Paturel R. (2000), « Externalisation et entrepreneuriat » In Verstraete T. (coord.), Histoire d'entreprendre, EMS, pp. $173-186$.

Paul M. (2004), L'accompagnement : une posture professionnelle spécifique, Paris, L'Harmattan.

Petrella F. et N. Richez-Battesti, (2010), « Régulation de la qualité dans les services à la personne en France : l'économie sociale et solidaire entre innovation et isomorphisme? » Management \& Avenir, 5 (35), pp. 273-292.

Plane J-M et O. Torres, (1998), « Le recours au conseil est-il un processus dénaturant pour la PME? », $4^{\text {ème }}$ CIFEPME, Nancy-Metz, Octobre, 22-24.

Pluchart J-J. (2014), « L'approche par les ressources des réseaux d'accompagnement entrepreneurial » Recherches en Sciences de Gestion, 3 (102), pp. 47-71. 
Pluchart J-J. (2013), « Les performances des réseaux d'accompagnement entrepreneurial » Vie \& sciences de l'entreprise, 1 (193), pp. 93-113.

Pouka Pouka M-R. et al., (2019), « L'influence de l'accompagnement entrepreneurial sur la performance de jeunes pme : une évaluation des structures camerounaises d'accompagnement » Revue Africaine de Management, 4 (2), pp. 36-59.

Randriamanalina T. (2020), « Perception de l'entrepreneuriat : le rôle de la famille » Revue Française d'Economie et de Gestion, 1 (3), pp. 47-63.

Roussel P. et F. Wacheux, (2005), Management des ressources humaines: Méthodes de recherche en sciences humaines et sociales, Bruxelles, De Boeck.

Ruževičius J. (2013), « Changements de paradigme du management de la qualité » International Business : Innovations, Psychology, Economics, 4 (1) (6), pp. 33-44.

Sammut S. (2003), «L'accompagnement des petites entreprises en création » In Marion S. et al., Réflexion sur les outils et les méthodes à l'usage du créateur d'entreprise, les Éditions de l'ADREG.

Theodoraki C. et K. Messeghem, (2016), « Écosystème de l'accompagnement entrepreneurial et stratégies coopétitives des incubateurs : Nouveaux défis stratégiques à relever " $X X V^{\text {ème }}$ Conférence de l'AIMS, Hammamet, 30 Mai-01 Juin.

Tounès A. (2003), L'intention entrepreneuriale : Une recherche comparative entre des étudiants suivant des formations en entrepreneuriat (bac+5) et des étudiants en DESS CAAE, Thèse de Doctorat en Sciences de Gestion, Université de Rouen.

Verzat C. et O. Toutain, (2015), « Former et accompagner des entrepreneurs potentiels, diktat ou défi ?»Savoirs, 3 (39), pp. 11-63.

Wilken P. (1979), Entrepreneurship: a comparative and historical study, Norwood, NJ, Ablex.

Wolff D. et T. Cuénoud, (2017), « Pour une approche renouvelée de l'accompagnement des créateurs et des repreneurs d'entreprise : le coaching entrepreneurial » Vie \& sciences de l'entreprise, 2 (204), pp. 146-163.

Yin R. (2003), Case study research: Design and methods, Thousand Oaks, Sage Publications, $3^{\text {th }}$ Edition.

Zghal R. (2004), « La recherche en entrepreneuriat : diversité d'approches et questions émergentes $» 4^{\text {th }}$ European Summer University, Paris, 24 Septembre - $1^{\text {er }}$ Octobre.

\section{NOTES}

1. Institut National de la Normalisation et de la Propriété Industrielle.

2. http://www.tunisieindustrie.nat.tn/fr/doc.asp?mcat=16\&mrub=182. 


\section{RÉSUMÉS}

L'accompagnement et la qualité constituent deux facteurs importants au lancement et à la survie des entreprises. Toutefois, peu d'études s'intéressent à ces deux facteurs conjointement. C'est dans ce contexte que s'inscrit cette contribution dont l'objectif est d'étudier, à travers une recherche exploratoire qualitative, l'apport des structures d'accompagnement à la création d'entreprises en matière de qualité auprès de douze entrepreneurs tunisiens en phase de précréation. Les résultats auxquels nous sommes parvenus montrent que les structures d'accompagnement jouent un rôle important dans l'adoption d'une démarche qualité par les entrepreneurs. Ces derniers ont trouvé dans l'INNORPI, une structure d'accompagnement vers la qualité, les prémisses de la réussite entrepreneuriale. En jouant la carte de la qualité, un choix stratégique proposé et soutenu par cette structure, les entrepreneurs espèrent garantir le succès et la survie de leurs projets.

Accompaniment and quality are two essential factors for the launch and survival of companies. However, few studies focus on these two factors together. Through qualitative exploratory research with twelve Tunisian entrepreneurs in the pre-creation phase, this contribution aims at studying how support structures contribute to the creation of companies in terms of quality. We show that support structures play a crucial role in helping entrepreneurs to adopt a quality approach. Entrepreneurs found in INNORPI an accompaniment structure towards quality premises of success. By playing the quality card proposed and supported by this structure as a strategic choice, entrepreneurs hope to guarantee the success and survival of their projects.

\section{INDEX}

Keywords : accompaniment, quality, entrepreneurs, pre-creation, INNORPI

Mots-clés : accompagnement, qualité, entrepreneurs, pré-création, INNORPI

Code JEL L26 - Entrepreneurship, L15 - Information and Product Quality • Standardization and Compatibility, M13 - New Firms • Startups, 034 - Intellectual Property and Intellectual Capital, O55 - Africa

\section{AUTEUR}

\section{SARHAN ABDENNADHER}

Maître-assistant à la FDS, Université de Sfax, Tunisie. 